\title{
Nondestructive estimation of modulus of rupture in a whole MDF panel
}

\author{
Mehran Roohnia
}

\begin{abstract}
A vibrational base nondestructive method is proposed to estimate the modulus of rupture (MOR) in medium density fiberboard (MDF) whole plate. A premium grade MDF panel was tested in longitudinal and flexural vibration approaches in panel longitudinal axis and its perpendicular direction. Then, the panel was cut into smaller and smaller plates and prismatic beams while the vibrational properties were collected in each step. Finally, the static bending method was used to evaluate the static MOE and MOR in final prismatic MDF beams. The dynamic and static MOEs were compared together and used along with the ogarithmic decrement to correlate with static MOR of the beams. A multiregression model of lumber MOR prediction was fitted also to MDF plate, considering a correction coefficient in terms of vibration plane (longitudinal or flexural). The modified multiregression model for the MDF panel was successful to estimate MOR, comparable to those of obtained in static bending standard methodology.
\end{abstract}

Keywords-Flexural; Longitudinal; MDF; Nondestructive; Rupture; Vibration

\section{Introduction}

A medium density fiber-board (MDF) panel, before reaching the market, must be assessed to check if it meets the standard qualifications or not. The conformity assessment costs are amplified if the panel is destroyed during the inspection. Thus, the inspection performance costs are far cheaper than the panel price. So, saving the MDF panel in conformity assessment procedure would be very affordable; however, all destructive inspections must be improved to nondestructive ones to complete this task. In practice, even in high quality researches, the wood base panel is normally subjected to destructive tests e.g. tensile, bending, water absorption, and dynamic mechanical analysis (Gabr et al., 2013) [1]. Among the destructive property assessments, listed in standard methodologies, the bending strength, namely, the modulus of rupture (MOR) is an important parameter.

In prismatic beams of $\mathrm{MDF}$, it is possible to estimate MOR, but when a beam is cut from a MDF panel, the destruction of the testing specimen is commenced; whether the test is destructive or not. For the dynamic modulus of elasticity determination from a whole panel longitudinal vibration, a promising argument was obtained in our

Mehran Roohnia

College of Agriculture and Natural Resources, Karaj Branch, Islamic Azad University

Iran fundamental investigations (Mirbolouk and Roohnia, 2015) [2]. It was shown that the panel could be analyzed in longitudinal vibration test the same as a beam, but considering a correction factor of the specimen width and Poisson's ratio suggested by Brancheriau (2011) [3]. Estimating the rupture (the end of the stress capacity) from vibrational properties which are set only on linear elastic section of the stress-strain curve, it seems to be a mission, hard to obtain. But fortunately, there are some encouraging influences of the sufficient correlations existing between the elastic and rupture moduli in previous literatures. To name a few, Bodig and Jayne (1993) provided a detailed chapter to discuss the good correlations between static MOE and MOR [3]. Halabe et al. (1997) showed that MOEs obtained through longitudinal or flexural vibration tests are correlated well with those of MORs in static bending test, but they did not report any acceptable argument to use ultrasonic velocity test for strength grading of wood [4]. Ayarkwa et al. (2001a) reported that the monitoring of acoustic emission is useful to predict MOR of finger-jointed wood [5]. They also obtained sufficiently good coefficients of determination to correlate the dynamic MOE of longitudinal vibration to MOR of the solid and finger-jointed specimens (2001b) [6]. Ross et al. (2005) reported a weak coefficient of determination, while comparing the dynamic MOE in stress wave test to MOR of the static bending test; however, the correlations between dynamic and static MOEs were good [7]. Lin et al. (2007) reported a strong correlation between MOR and dynamic modulus of elasticity (MOE), while studying the changes in dynamic MOE and bending properties of railroad ties in Taiwan [8]. After all, the coefficient of determination between MOE and MOR is good enough to be extended into international standard methodologies. For an example, timber grading according to EN standard no. 338 formulates the evaluation of MOEs. The modulus of rupture is predicted through a provided table in terms of the timber grade, the density, and the modulus of elasticity varieties. For MDF which is more homogeneous than solid wood, a similar scenario is expected. That's why the static and dynamic elastic moduli are compared together in this paper along with the modulus of rupture.

Despite the good correlation, MOE must not be the lonely parameter to affect the modulus of rupture. When a piece of material is pushed to be deformed, the failure occurs as the external force overcomes the internal bonding forces. The molecules are sliding one on another, and the crack occurs after overcoming the internal friction. Based on this hypothesis, to predict a more reliable modulus of rupture, the internal friction must be taken into account. Damping (logarithmic decrement) is a good indicator for the internal friction (Tsoumis, 1991) [9] and might be useful to estimate MOR, but undeniably in a combination with dynamic 
modulus of elasticity. Bodig and Jayne (1993) [3] reported a higher coefficient of determination when the combination of elasticity and internal friction was utilized for modulus of rupture estimation. For wood, Divos et al. (2010) [10] provided a multi regression equation to estimate the bending strength, taking into the account the dynamic elastic modulus and logarithmic decrement of flexural vibration as well as the effects of knots in marginal and total area within the lumber:

\section{$\mathrm{MOP}=29.36+3.071 \mathrm{MOE}-0.5778 \delta$}

Where $\delta$ is the logarithmic decrement, MOR is the modulus of rupture in MPa, and MOE is the modulus of elasticity in GPa. There were also parameters due to the knot area within the lumber (Divos and Tanaka, 1997) [11] which are kept affectless in MDF approach.

A revision on Divos's suggested multi regression model, considering the dynamic modulus of elasticity obtained from longitudinal or flexural vibration of the beams and the whole panel, is noted here. Though, the equality between internal frictions obtained from the panel and their final beams were not reported in our previous paper (Mirbolouk and Roohnia, 2015) [2]; the following approach is to study if a combination of modulus of elasticity and internal friction is the solution for estimating the MOR of a whole panel or not.

\section{Materials and Methods}

\section{A. Materials}

Following the procedure introduced in our previous paper (Mirbolouk and Roohnia, 2015) [2], a premium grade MDF panel (made in Iran) which is clear without any visual defect with nominal (length $\times$ width $\times$ thickness) dimensions of 244 $\times 122 \times 1.6 \mathrm{~cm} 3$ was selected. The panel was cut consecutively in three steps into four plates of $100 \times 60 \times$ $1.6 \mathrm{~cm} 3$, then 16 smaller plates of $50 \times 30 \times 1.6 \mathrm{~cm} 3$ and finally 64 prismatic beams of $4.5 \mathrm{~cm}$ wide oriented in panel direction (D) and its perpendicular direction (PD).

The specimens were kept in a climatic chamber $(65 \% \mathrm{RH}$, $21 \pm 1^{\circ} \mathrm{C}$ ) for a period until the moisture content was stabilized nominally at $12 \%$ of the moisture content.

\section{B. Methods}

The NDTLAB ${ }^{\circledR}$ portable system for flexural vibration (Roohnia, 2006 and 2007) [12,13] and its newer release for longitudinal vibration tests, LSTRESS (Roohnia et al., 2011) [4], was used for acoustic analyses. The plates and beams were knocked using a small hammer or a light steel ball to be excited in flexural or longitudinal vibrations in both $\mathrm{D}$ and PD directions; they were recorded by a unidirectional microphone. To simulate the free-free beam or plate, the specimens lean on a soft thin rubber from their nodal points. The specimen is excited from an end, and the vibration sound is recorded from the other end by a unidirectional microphone. The sampling rate of recording software was $44100 \mathrm{HZ}$ with a frequency resolution close to $3 \mathrm{~Hz}$. The $1 \mathrm{st}$ modal frequency is evaluated using FFT analysis.

For the longitudinal vibration test:

$V=2 L f$

$E_{L}=\rho V^{2}$

$f=\frac{1}{2 L} \sqrt{\frac{E_{L}}{\rho . C}}, \quad C=1+\frac{\pi^{2}}{A L^{2}}\left(I_{Z} v_{x y}^{2}+I_{y} v_{x z}^{2}\right)$

where $\mathrm{V}$ is sound velocity $(\mathrm{m} / \mathrm{s}), \mathrm{L}$ is sound traveling distance (specimen length, $\mathrm{m}$ ), and $\mathrm{f}$ is frequency of the $1^{\mathrm{st}}$ mode of longitudinal vibration $(\mathrm{Hz}) . \mathrm{E}_{\mathrm{L}}$ is the longitudinal dynamic modulus of elasticity $(\mathrm{Pa})$, and $\rho$ is density $\left(\mathrm{kg} / \mathrm{m}^{3}\right)$. Differences in cross-sectional dimensions may create different values of error in modal evaluations, so, $\mathrm{C}$ is Brancheriau's correction coefficient (Brancheriau, 2011; Mirbolouk and Roohnia, 2015) [2,15] for the dynamic modulus of elasticity, A is cross section area $\left(\mathrm{m}^{2}\right), \mathrm{I}$ is the moment of inertia $\left(\mathrm{m}^{4}\right)$, and $v$ is Poisson's ratio (with regards to grain coordinates).

For flexural vibration test, the Euler-Bernoulli's elementary theory was used:

$E_{f}=\frac{48 \pi^{2} f^{2} \rho L^{4}}{m_{1}{ }^{4} t^{2}}$

Where $\mathrm{E}_{f}$ is the axial modulus of elasticity obtained in flexural vibration test $(\mathrm{Pa}), \mathrm{t}$ is the thickness $(\mathrm{m})$, and $\mathrm{m}_{1}$ is a constant depending on the boundary condition and the mode number. In this particular approach for the $1^{\text {st }}$ mode of a both ends free condition, $\mathrm{m}_{1}$ is equal to 4.73 (Bodig and Jayne, 1993).

In either the longitudinal or the flexural vibration tests, the logarithmic decrement $\delta$, as an indication for internal friction, is calculated using the attenuation curve in temporal field following Bodig and Jayne (1993) and Brémaud (2008) $[3,16]$ :

$$
\delta=\frac{1}{n} \ln \left(\frac{x_{0}}{x_{n}}\right)
$$

where $X_{0}$ is the greater than the two amplitudes, and $X_{n}$ is the amplitude of a peak, $n$ periods away (vibration associated with the first mode).

Then, in a Zwick/Roell universal testing machine, the specimens leaned on round hard supports with a span of $38 \mathrm{~cm}$ 
and were subjected to a three point static bending test (regarding to ASTM D1037-06) to calculate the static modulus of elasticity and the modulus of rupture:

$$
M O E=\frac{L^{3}}{4 b t^{3}} \frac{\Delta F}{\Delta y}
$$

$M O R=\frac{3 F_{\max } L}{2 b t^{2}}$

In which, $\Delta \mathrm{F} / \Delta \mathrm{y}$ is the slope of the straight line portion of the load-deflection curve $(\mathrm{N} / \mathrm{m}), \mathrm{b}$ is the width of the specimen $(\mathrm{m})$, and $\mathrm{F}_{\max }$ is the maximum load at rupture point $(\mathrm{N})$.

While the obtained moduli of elasticity in these three different test methods are compared, MOR from the multi regression model (Eq. 1) is plotted and revised for MDF versus the measured MOR in static bending test (Eq. 8).

\section{Results and Discussion}

The final prismatic beams are demonstrated in terms of the moduli of elasticity in three different varieties of static or dynamic methodologies in Figure 1. Comparing all the methodologies, the coefficient of determinations was sufficiently high, while the static bending test and the longitudinal vibration methods' results were more similar. The positive shifts in flexural vibration results might be related to the viscoelastic behavior of MDFs and proper delay in flexural deflection in high speed vibrating systems; however, the old story of the reasons for these variations among the static and the dynamic methods are out of the present approach's scope.

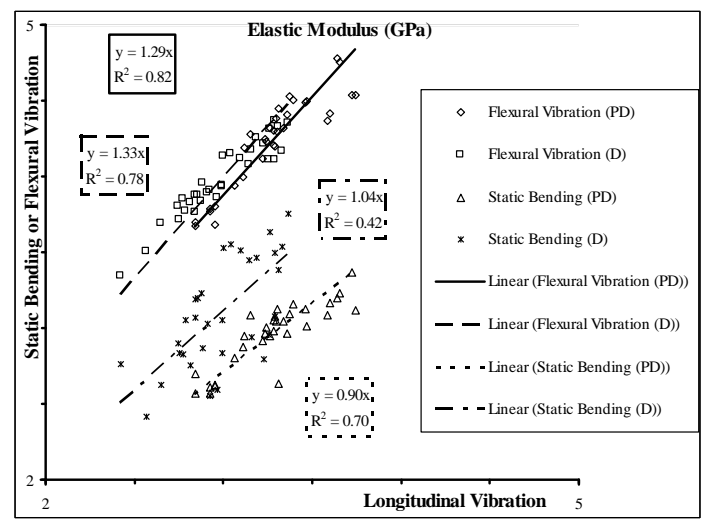

Fig. 1 A comparison in brief among the three test methods in term of modulus of elasticity in panel direction (D) and its perpendicular (PD)

The static and dynamic moduli were correlated to the static modulus of rupture in Figure 2. Both the dynamic moduli were highly correlated to the rupture, but the coefficient of determination of static modulus of elasticity in comparison with the modulus of rupture was weak. So, continuing the approach to reach a reliable way to predict the rupture point of MDF beams from nondestructive vibration data, either the longitudinal or the flexural vibration, turned to be promising.

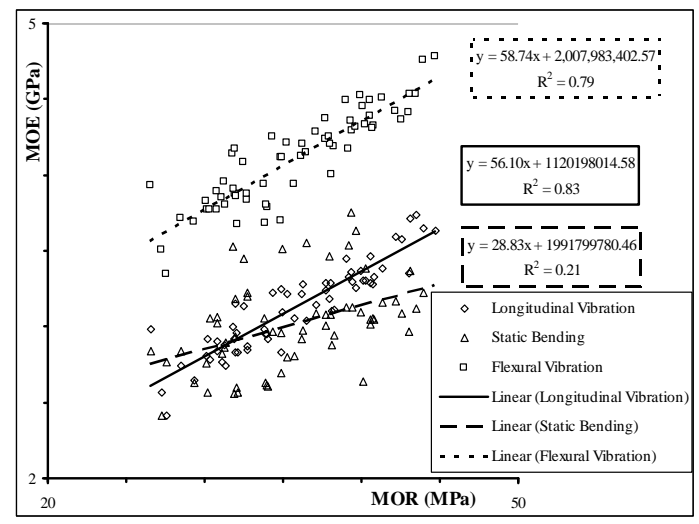

Fig. 2 Modulus of elasticity vs. modulus of rupture in three different approaches

In Figure 3, the combination of the modulus of elasticity and the internal friction index $(\mathrm{MOE} / \delta)$ are indicated versus the modulus of rupture. Regarding the coefficients of determination, the flexural vibration was more meaningful looking to the estimated modulus of rupture in studied MDF prismatic beams.

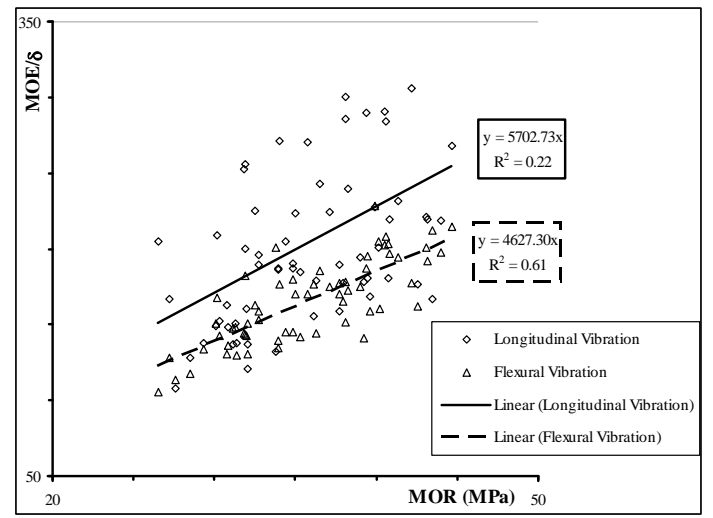

Fig. 3 A combination of the modulus of elasticity and the logarithmic decrement $(M O E / \delta)$ in two different vibration tests, vs. modulus of rupture.

The suggested multi regression model (Eq. 1) also considers both the internal friction and the modulus of elasticity. Figure 4 shows the predicted MOR values in longitudinal and flexural vibration versus their experimental static values. The strong correlation observed in this figure is very promising, but it is suffering from an invalid intercept. The intercept of the trends is invalid because the prediction must also approach zero as the experimental MOR value is heading to zero. So, the multi regression model must have a new intercept to compensate for false deviations in the combination mode. A new intercept was developed, but the slope of trend was changed to reasonable predicted data. The 
modifications began in flexural vibration data, then considering the differences between flexural and longitudinal moduli of elasticity, the longitudinal one was raised 1.3 times to develop a newer multi regression model for the studied specimens of MDF.

$$
\mathrm{MOP}=4.8 \alpha^{2}(-5+3.071 \mathrm{MOE}-0.5778 \delta)
$$

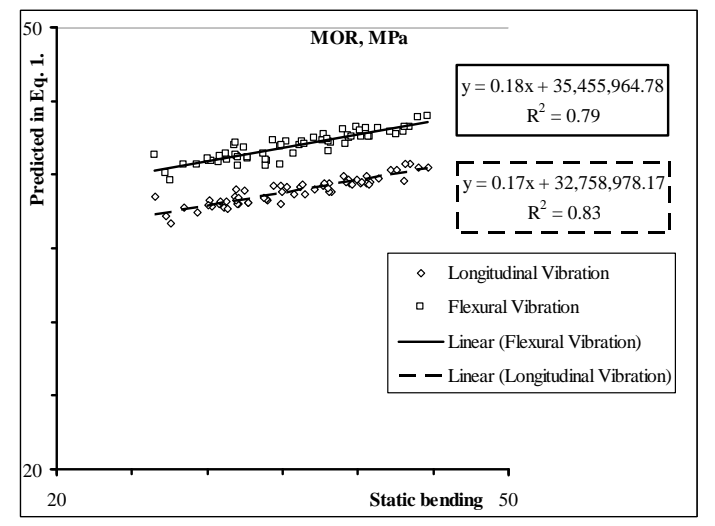

Fig. 4 Modulus of rupture predicted in Eq 1. vs. modulus of rupture in static bending tests

Where, $a$ is a constant due to the vibration plane. For flexural vibration, a is ignored in flexural vibration, but it is equal to 1.3 in longitudinal vibration. MOR, MOE, and $\delta$ are the same as they were defined before. Figure 5 shows the new estimation model (Eq. 9) versus the experimental static modulus of rupture.

Regarding what is seen in Figure 5, the new model was successful, either in the longitudinal or the flexural vibration tests to estimate the modulus of rupture of the studied beam specimens, oriented in panel direction or its perpendicular direction.

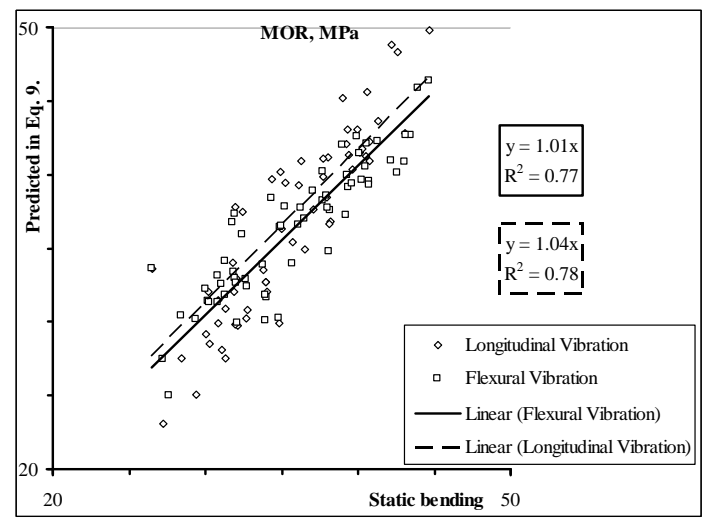

Fig. 5 Modulus of rupture predicted in new model (Eq 9.) vs. modulus of rupture in static bending tests
The similarity of the moduli of elasticity obtained in whole panel or the prismatic beam vibration is already certified by Mirbolouk and Roohnia (2015) [2] for longitudinal vibration test. But, for the flexural vibration, the modal FFT in big whole MDF plate could not be analyzed. The frequency reading interval used in the FFT analyzer did not sufficiently cover the low frequency of the 1st mode of flexural vibration in the big whole plate. So, the average of the 1st flexural frequencies of four smaller plates (with higher natural frequencies) was used.

The modulus of rupture was estimated again using the modulus of elasticity and logarithmic decrements of both the longitudinal and flexural vibration of MDF plates, compared to the actual modulus of rupture, averaged in repeated prismatic beams. Figure 9 shows that the new multi regression model was successful enough, for both the longitudinal and flexural vibration, to estimate the modulus of rupture. In panel perpendicular direction, sufficiently acceptable estimations were not considered (Figure 10).

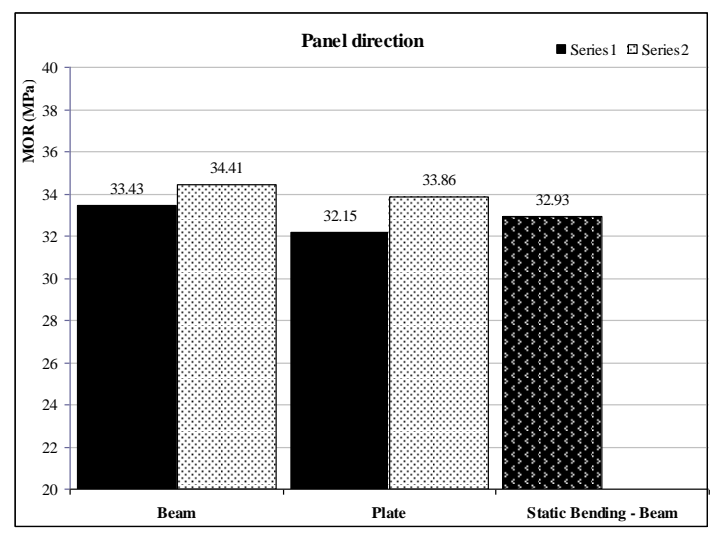

Fig. 9 Modulus of rupture in panel direction predicted in plate vibration compared to the beam vibration and the static bending tests. Series 1: Longitudinal, Series 2: Flexural

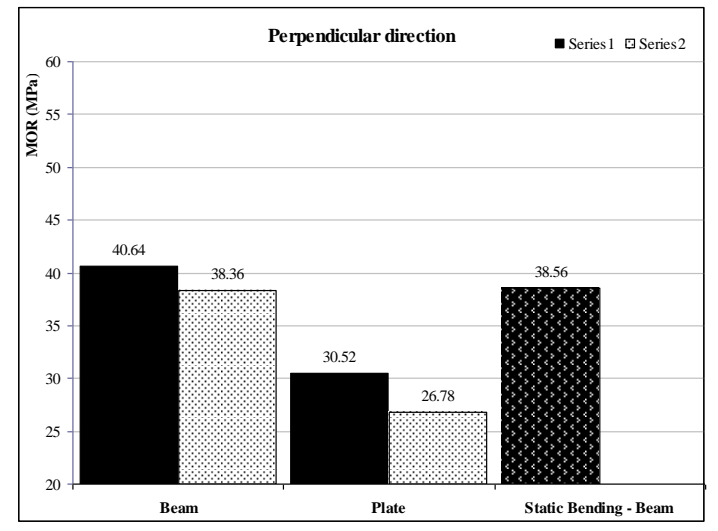

Fig. 10 Modulus of rupture in panel perpendicular direction predicted in plate vibration compared to the beam vibration and the static bending tests. Series 1: Longitudinal, Series 2: Flexural 


\section{Conclusion}

A nondestructive suggestion was proposed and studied to estimate the modulus of rupture in whole plate of the medium density fiber board. The methodology for beam rupture estimations was improved to assess the panel in either flexural or longitudinal vibrations. The proposed methodology was very promising.

- Regardless of the known causes of shift in modulus of elasticity values (e.g. the viscoelastic properties or the deflection delay and loading speeds; shear deflection and rotary inertia in bending and so on) in either the vibration or the static bending, there were sufficient correlation coefficients between the methods to evaluate the moduli of elasticity of a MDF prismatic beam.

- The vibrational elastic moduli (both the flexural and the longitudinal) were correlated to the modulus of rupture, better than the static bending elastic modulus.

- There was not any sufficient correlation between the logarithmic decrement and the modulus of rupture at least for the longitudinal vibration test.

- The combination of the vibrational elastic moduli and the logarithmic decrements $(\mathrm{MOE} / \delta)$ was more encouraging to estimate the modulus of rupture in the flexural vibration test.

- The multi regression model for estimating the modulus of rupture of the solid timber from the elastic modulus and the logarithmic decrement was successful for MDF, but a correction coefficient other that the effect of timber knots was developed and inserted into the equation in terms of the methods of the vibration test (longitudinal or flexural).

- The developed equation was also successful to predict the modulus of rupture from the vibration of the whole MDF plate, in panel direction, though the panel perpendicular direction still needs some more investigations to be clearly understood.

\section{References}

[1] M. H. Gabr, N. T. Phong, M. A. Abdelkareem, K. Okubu, K. Uzawa, I. Kimpara, T. Fujii, Mechanical, thermal and moisture absorption properties of nano-clay reinforced nano-cellulose, biocomposites, Cellulose, 2013, Vol. 20. NO. 2, pp.819-826.

[2] P. Mirbolouk, M. Roohnia, Evaluation of dynamic modulus of elasticity of medium density fiberboard panel from longitudinal vibration tests on specimens, BioResources, 2015, vol. 10, pp. 613-621.
[3] J. Bodig, B.A. Jayne, (1993) Mechanics of Wood and Wood Composites. Krieger publishing company, Malabar, Florida, 1993.

[4] U. B. Halabe, G.M. Bidigalu, H.V.S. Gangarao, R. Ross. (1997). Nondestructive evaluation of green wood using stress wave and transverse vibration techniques, Materials and Evaluations, 1997, Vol. 55. No. 9, pp. 1013-1018.

[5] J. Ayarkwa, Y. Hirashima, K. Ando, Y. Sasaki Y, Monitoring acoustic emissins to predict modulus of rupture of finger-joints from tropical African hardwoods, Wood and Fiber Science, 2001a, Vol. 33. No. 3. pp.450-464.

[6] J. Ayarkwa, Y. Hirashima, Y. Sasaki Y, Predicting modulus of rupture of solid and finger jointed tropical African hardwoods using longitudinal vibration. Forest Prod. J, 2001b, No. 51, pp. 85-92.

[7] R. Ross, J. Zerbe, X. Wang, D. Green, R. Pellerin, Stress wave nondestructive evaluation of Douglas-fir peeler cores, For. Prod. J, Vol. 55, No. 3, pp. 90-94.

[8] C. J. Lin, T.H. Yang, D. Z. Zhang, S. Y. Wang, F.C. Lin, Changes in the dynamic modulus of elasticity and bending properties of railroad ties after 20 years of service in Taiwan, Building and environment, 2007, No. 42. No. 3, pp. 1250-1256.

[9] G. Tsoumis G, Science and technology of wood, Van Nostrand Reinold, 1991, pp. 204-207.

[10] F. Divos, A.S. Kiss, "Strength grading of structural lumber by portable lumber grading - effect of knots". In Proceedings of the future of quality control for wood and wood products, Edinburgh, Great Britain, May 2010.

[11] F. Divos, T. Tanaka, Lumber strength estimation by multiple regression, Holzforschung, 1997, Vol. 51. No.5, pp. 467-471.

[12] M. Roohnia, I. Bremaud, D. Guibal, N. Manouchehri, "NDT-LAB; Software to evaluate the mechanical properties of wood. Pages 213-218, In: Proc International Conference on Integrated Approach to Wood Structure Behaviour and Applications Joint meeting of ESWM and Cost Action E35", Fioravanti, M, Macchioni, N. (eds.), Florence Italy, DISTAF - University of Florence, May 2007.

[13] M. Roohnia, (2007). NDT-LAB; System to evaluate the mechanical properties of wood. IR-Patent No. 44032/22-08-1386, Iranian official Journal, 2007, Available at: http://www.gazette.ir/Detail.asp?NewsID=914760224197351\&paperI $D=975266637116773$ accessed: $2015-03-13$.

[14] M. Roohnia, S. F. Hashemi-dizaji, L. Brancheriau, A. Tajdini, A. H. Hemmasi, N. Manouchehri, Effect of soaking process in water on the acoustical quality of wood for traditional musical instruments, BioResources, 2011, Vol. 6. No. 2, pp. 2055-2065.

[15] L. Brancheriau, Corrections for Poisson effect in longitudinal vibrations and shearing deformations in transverse vibrations applied to a prismatic orthotropic body. in Mechanical Vibrations: Types, Testing and Analysis, Galloway, A. L. (ed.), Nova Science Publishers, ISBN-13: 978-1616682170, 2011, pp. 205-224.

[16] I, Brémaud, "Caractérisation mécanique des bois et facture : origines et recensement de la variabilité. Actes de la journée d'étude Le bois : instrument du patrimoine musical - Cité de la Musique”. May 2008, pp. $24-46$. 